\title{
Photoelectrochemical measurement of effective diffusion length of carriers in lamellar semiconductors. Application to InSe
}

\author{
B. Theys \\ Laboratoire de Physique des Solides, CNRS, 1 place Aristide Briand, 92195 Meudon Cedex, France
}

(Reçu le $1^{\text {er }}$ septembre 1987, révisé le 21 décembre 1987, accepté le 25 avril 1988)

\begin{abstract}
Résumé. - A cause de l'anisotropie des propriétés optiques et électroniques des matériaux lamellaires, la densité de photocourant (ou le rendement quantique) débité par une jonction semiconducteur lamellaire/électrolyte dépend à la fois de l'angle d'incidence et de l'angle de polarisation du faisceau lumineux par rapport aux axes cristallographiques de l'électrode. Une description analytique de cette dépendance est développée et on montre que ce résultat peut être exploité pour mesurer, dans cette famille de matériaux, la longueur de diffusion effective des porteurs minoritaires photocréés. Dans une seconde partie, cette méthode de mesure est appliquée au composé III-VI InSe. Les conclusions sont tout à fait en accord avec les valeurs de longueurs de diffusion publiées précédemment.
\end{abstract}

\begin{abstract}
Owing to the anisotropy of the optical and electronic properties of layered materials, the photocurrent density (or the quantum efficiency) delivered by a lamellar semiconductor/electrolyte junction is dependent upon both the angle of incidence and the angle of polarization of the incoming light beam with respect to the crystallographic axis of the electrode. This dependence is analytically described and it is shown that this result can be used to measure the photogenerated minority carrier effective diffusion length in this family of materials. In a second part, this method of measurement is applied to the III-VI compound InSe. The conclusions agree very well with the values of diffusion lengths published previously.
\end{abstract}

\section{Introduction.}

It is well known that the effective diffusion length of photogenerated minority carriers is a key parameter in the evaluation of the quantum or energetic efficiency of photovoltaic devices. Different techniques have been proposed to measure such a diffusion length. Among them, surface photovoltage (S.P.V.) [1-3], photoresponse [4-6] and light beam induced current (L.B.I.C.) [7] are the most commonly used. With the development of photoelectrochemistry (P.E.C.), techniques taking advantage of the rectifying properties of the electrolyte-semiconductor junctions have also been developed more recently. The preparation of such junctions does not necessitate complicated technology or high-temperature processes which may modify the intrinsic properties of the materials under investigation. The S.P.V. [8-9] as well at the photoresponse [10] or
L.B.I.C. [11] methods have been used with such liquid contacts.

The properties of lamellar materials are strongly influenced by their layered structure. Reference [12] gives a large survey of the basic chemical and physical characteristics of this family of solids. From a photoelectrochemical point of view, a great number of studies were reported in the last ten years [13-18]. It appears that the rectifying character of the electrode/electrolyte junction and the anisotropy of the semiconducting electrode can both be exploited together in order to measure unknown properties of the semiconductor. For example, such arguments, but based on a restrictive theory, were applied to measure the optical anisotropy of some transition metal dichalcogenides [19]. In the present paper, we present a technique for measuring the effective diffusion length of photogenerated minority carriers in a lamellar semiconductor, both parallel and 
perpendicular to the $c$ axis of the crystal. One of the major advantages of this technique is that it is based upon the measurement of ratios and does not necessitate the knowledge of absolute values which are always difficult to determine precisely.

After a theoretical and detailed description of the technique, we present and discuss the results obtained with InSe. This material is a layered compound in which the electronic transport parameters and optical properties are very anisotropic [20, 21].

\section{Theory.}

When a semiconductor-electrolyte junction is illuminated, a photocurrent flows. If the band bending inside the solid is large enough, recombination of carriers can be neglected close to the interface, and the so-called Gärtner model [22] can be used to calculate the intensity of this photocurrent. For a given incident wavelength and a given applied voltage, it appears then that this current is a function of 5 parameters : the photon flux (which we will consider in the following to be equal to unity because we are dealing with ratios), the reflection coefficient of the incoming light, the optical absorption coefficient, the space-charge width inside the electrode and, finally, the effective diffusion length of the carriers.

Lamellar materials are anisotropic and, optically speaking, they are uniaxial. The optical axis is parallel to the $c$ axis of the crystal [23]. Therefore the photocurrent will depend upon $: \mathrm{i}$ ) the orientation of the solid/liquid interface with respect to the $c$ axis of the crystal, ii) the polarization of the incoming light with respect to this $c$ axis and iii) the angle of incidence of the light beam.

Two types of electrodes will be considered: parallel (//) and perpendicular $(\perp)$ to the $c$ axis. // electrodes have an active surface (in contact with the electrolyte) which is normal to the layers and which is made up of the layer edges (Fig. 1). $\perp c$ electrodes have an active surface which is parallel to the layers and which is thus made up of a Van der Waals (or cleavage) plane.

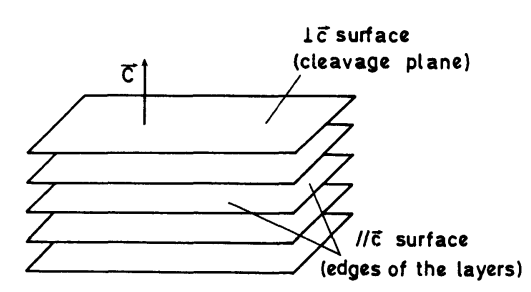

Fig. 1. - Definition of $/ /$ and $\perp c$ surfaces.

1.1 // $c$ ELECTRODE. - For this orientation we simply have to consider the normal incidence for the incoming light because sufficient information can be obtained in such a configuration.
We define the $\mathrm{O} z$ direction as the one of the optical axis of the lamellar material (the $c$ axis). The incident beam being normal to the liquid/solid interface, the wave vectors of the refracted beams are in the $\mathrm{O} y$ direction. The complex refractive index $\tilde{n}_{x}$ is associated with the ordinary beam (electric field in the $\mathrm{O} x$ direction) and $\tilde{n}_{z}$ is associated with the extraordinary beam (electric field in the $\mathrm{O} z$ direction). We suppose that both $\tilde{n}_{x}=\left(n_{x}+\right.$ $\left.i \lambda \alpha_{x}(4 \pi)^{-1}\right)$ and $\tilde{n}_{z}=\left(n_{z}+i \lambda \alpha_{z}(4 \pi)^{-1}\right)$ are known ( $\alpha$ is the absorption coefficient and $\lambda$ is the wavelength of the radiation in vacuum).

From an experimental point of view, each one of these two beams can be obtained separately by illuminating the junction with a linearly polarized light $(E / /$ or $\perp c)$ and the ratio of the two corresponding photocurrents can be measured.

On a theoretical basis, both of the photocurrents can be estimated using the Gärtner model [22], as previously mentioned. According to this model we have (per unit of light flux) :

- for the ordinary beam

$$
J_{\mathrm{o}}=-q\left(1-R_{\mathrm{o}}\right)\left[1-\frac{\exp \left(-\alpha_{x} W\right)}{1+\alpha_{x} L_{\perp}}\right]
$$

- for the extraordinary beam

$$
J_{\mathrm{e}}=-q\left(1-R_{\mathrm{e}}\right)\left[1-\frac{\exp \left(-\alpha_{z} W\right)}{1+\alpha_{z} L_{\perp}}\right] \text {. }
$$

$q$ is the elementary charge, $W$ the space charge width and $L_{\perp}$ is the effective diffusion length of the photogenerated carriers in a direction parallel to the layers (unknown). $R_{\mathrm{o}}$ and $R_{\mathrm{e}}$ are the reflection coefficients of the ordinary and extraordinary beams respectively. They can be calculated using the Fresnel formula [24]:

$$
R_{\mathrm{o}}=\frac{\left(n_{x}-n_{\mathrm{el}}\right)^{2}+\left(\alpha_{x}^{2} \lambda^{2} / 16 \pi^{2}\right)}{\left(n_{x}+n_{\mathrm{el}}\right)^{2}+\left(\alpha_{x}^{2} \lambda^{2} / 16 \pi^{2}\right)}
$$

and

$$
R_{\mathrm{e}}=\frac{\left(n_{z}-n_{\mathrm{el}}\right)^{2}+\left(\alpha_{z}^{2} \lambda^{2} / 16 \pi^{2}\right)}{\left(n_{z}+n_{\mathrm{el}}\right)^{2}+\left(\alpha_{z}^{2} \lambda^{2} / 16 \pi^{2}\right)}
$$

where $n_{\mathrm{el}}$ is the refractive index of the transparent electrolyte.

Finally, the ratio $J_{\mathrm{e}} / J_{\mathrm{o}}$ can easily be calculated with the help of a desktop computer for different values of the wavelength of the incoming light. Such spectra $J_{\mathrm{e}} / J_{\mathrm{o}}(\lambda)$ can be obtained for different values of $L_{\perp}$ and theoretical and experimental curves are compared in order to obtain the diffusion length of photogenerated carriers perpendicular to the $c$ axis in the sample under investigation. 
1.2 $\perp c$ ELECTRODES. - Under normal incidence, there exists only one refracted beam (the ordinary one) into the semiconductor as it can be seen by considering that $E$ is always normal to $c$.

Consequently, to compare the effects of the ordinary and of the extraordinary beams it is necessary to use an oblique incidence. For such conditions, it is possible to solve the Maxwell equations exactly in an absorbing uniaxial crystal [25]. It is rather tedious and the properties of semiconductors allow approximations which make the problem much simpler. First, for photon energy close to the band gap width, the inequality $\alpha^{2} \lambda^{2} / 16 \pi^{2} n^{2} \ll 1$ is fulfilled. It results that the real part of the refractive index of the semiconductor can be approximated by the (real) refractive index of a transparent medium [26]. Furthermore, we will consider that both the ordinary and the extraordinary beams are linearly polarized [24]. The problem with which we have to deal is then similar to the one of a transparent uniaxial medium except that the amplitudes of the vibrations decay exponentially with the penetration depth inside the solid.
1.2.1 Concerning the ordinary beam. - The vibrations of that beam are normal to the optical axis $\mathrm{O} z$. The refractive index (real) $n_{x}$ and the absorption coefficient $\alpha_{x}$ are associated with it. Its index surface is spherical (thus isotropic) and, consequently, the angle of refraction follows Descartes'law. If $\theta$ is the angle of incidence (with respect to $\mathrm{O} z$ ) and $\theta_{0}$ the angle of refraction, the following relationship is satisfied :

$$
n_{\mathrm{el}} \sin \theta=n_{x} \sin \theta_{0} .
$$

For a unit photon flux, the corresponding photocurrent can be written as :

$$
\begin{aligned}
J_{\mathrm{o}}=- & q\left(1-R_{0}\right) \times \\
\times & {\left[1-\frac{\exp \left(-\alpha_{x} W / \cos \theta_{0}\right)}{1+\left(\alpha_{x} L_{\|} / \cos \theta_{0}\right)}\right] \frac{\cos \theta}{\cos \theta_{0}} . }
\end{aligned}
$$

$L_{\|}$is the unknown diffusion length perpendicular to the layers $(/ / c)$. The absorption coefficient is multiplied by $\left(\cos \theta_{0}\right)^{-1}$ because of the oblique incidence. $R_{0}$ is the reflectivity of the ordinary beam, and it can be obtained from the Drude formula [27] :

$$
R_{0}=\frac{\left[\cos \theta-\operatorname{Re}\left(N_{x}^{\prime 2}-\sin ^{2} \theta\right)^{1 / 2}\right]^{2}+\left[\Im \mathrm{m}\left(N_{x}^{\prime 2}-\sin ^{2} \theta\right)^{1 / 2}\right]^{2}}{\left[\cos \theta+\operatorname{Re}\left(N_{x}^{\prime 2}-\sin ^{2} \theta\right)^{1 / 2}\right]^{2}+\left[\jmath \mathrm{m}\left(N_{x}^{\prime 2}-\sin ^{2} \theta\right)^{1 / 2}\right]^{2}}
$$

with

$$
N_{x}^{\prime}=\frac{n_{x}}{n_{\mathrm{el}}}+i \frac{\alpha_{x} \lambda}{4 \pi n_{\mathrm{el}}}=n_{x}^{\prime}+i k_{x}^{\prime}
$$

Real and imaginary parts which appear in (5) are given by

$$
\begin{aligned}
& \operatorname{Re}\left(N_{x}^{\prime 2}-\sin ^{2} \theta\right)^{1 / 2}=\left\{\frac{1}{2}\left[\left(n_{x}^{\prime 2}-\sin ^{2} \theta\right)^{2}+4 n_{x}^{\prime 2} k_{x}^{\prime 2}\right]^{1 / 2}+\frac{1}{2}\left(n_{x}^{\prime 2}-\sin ^{2} \theta\right)\right\}^{1 / 2} \\
& \operatorname{Im}\left(N_{x}^{\prime 2}-\sin ^{2} \theta\right)^{1 / 2}=\left\{\frac{1}{2}\left[\left(n_{x}^{\prime 2}-\sin ^{2} \theta\right)^{2}+4 n_{x}^{\prime 2} k_{x}^{\prime 2}\right]^{1 / 2}-\frac{1}{2}\left(n_{x}^{\prime 2}-\sin ^{2} \theta\right)\right\}^{1 / 2} .
\end{aligned}
$$

1.2.2 Concerning the extraordinary beam. - The refractive index and absorption coefficient associated with that beam are not isotropic and their values depend upon the direction of propagation.

First, it is necessary to keep in mind that the wave normal and the ray are not parallel. The refracted energy propagates along the ray direction. If $\theta_{\mathrm{er}}$ is the refraction angle of this energy with respect to $\mathrm{O} z$, it is defined by [28] :

$$
\operatorname{tg} \theta_{\mathrm{er}}=\frac{n_{x}}{n_{z}} \frac{n_{\mathrm{el}} \sin \theta}{\left(n_{z}^{2}-n_{\mathrm{el}}^{2} \sin ^{2} \theta\right)^{1 / 2}}
$$

in which $\theta$ is the angle of incidence. REVUE DE PHYSIQUE APPLIQUÉE. - T. 23, N 8, AOUTT 1988
The angle of refraction $\theta_{\mathrm{en}}$ of the wave normal does obey the Descartes law [24] :

$$
n_{\mathrm{el}} \sin \theta=n_{\mathrm{e}} \sin \theta_{\mathrm{en}}
$$

where

$$
n_{\mathrm{e}}=\frac{n_{x} n_{z}}{n_{z}^{2} \cos ^{2} \theta_{\mathrm{en}}+n_{x}^{2} \sin ^{2} \theta_{\mathrm{en}}}
$$

(9) and (10) give

$$
\cos ^{2} \theta_{\mathrm{en}}=\frac{n_{z}^{2}-n_{\mathrm{el}}^{2} \sin ^{2} \theta}{n_{z}^{2}+n_{\mathrm{el}}^{2} \sin ^{2} \theta\left[\frac{n_{z}^{2}}{n_{x}^{2}}-1\right]} .
$$


The absorption coefficient also depends upon $\theta_{\text {en }}$ :

$$
\alpha_{\mathrm{e}}=\frac{n_{\mathrm{e}}^{2}}{n_{x}^{2}} \alpha_{x} \cos ^{2} \theta_{\mathrm{en}}+\frac{n_{\mathrm{e}}^{2}}{n_{z}^{2}} \alpha_{z} \sin ^{2} \theta_{\mathrm{en}} .
$$

For a given angle of incidence $\theta$, the photocurrent $J_{\mathrm{e}}$ generated by the absorption of the photons of a monochromatic extraordinary beam can be expressed. For a unit flux this gives :

$$
\begin{aligned}
J_{\mathrm{c}}= & -q\left(1-R_{\mathrm{e}}\right) \times \\
& \times\left[1-\frac{\exp \left(-\alpha_{\mathrm{e}} W / \cos \theta_{\mathrm{er}}\right)}{\left[1+\left(\alpha_{\mathrm{e}} L_{\|} / \cos \theta_{\mathrm{er}}\right)\right]}\right] \frac{\cos \theta}{\cos \theta_{\mathrm{er}}}
\end{aligned}
$$

$R_{\mathrm{e}}$ is the reflectivity of the extraordinary beam at the electrolyte/electrode interface. It can be obtained from the Drude formula [27] :

$$
R_{\mathrm{e}}=\frac{\left[n_{x}^{\prime} n_{z}^{\prime} \cos \theta-\mathcal{R e}\left(N_{z}^{\prime 2}-\sin ^{2} \theta\right)^{1 / 2}\right]^{2}+\left[\left(n_{x}^{\prime} k_{z}^{\prime}+n_{z}^{\prime} k_{x}^{\prime}\right) \cos \theta-\operatorname{Im}\left(N_{z}^{\prime 2}-\sin ^{2} \theta\right)^{1 / 2}\right]^{2}}{\left[n_{x}^{\prime} n_{z}^{\prime} \cos \theta+\operatorname{Re}\left(N_{z}^{\prime 2}-\sin ^{2} \theta\right)^{1 / 2}\right]^{2}+\left[\left(n_{x}^{\prime} k_{z}^{\prime}+n_{z}^{\prime} k_{x}^{\prime}\right) \cos \theta+\operatorname{Im}\left(N_{z}^{\prime 2}-\sin ^{2} \theta\right)^{1 / 2}\right]^{2}}
$$

Symbols have the same meanings as those defined by relations (6), (7a) and (7b), inverting $x$ and $z$ subscripts where appropriate.

Finally, $J_{\mathrm{e}}, J_{\mathrm{o}}$ and the ratio $J_{\mathrm{e}} / J_{\mathrm{o}}$ can be calculated for different wavelengths and the theoretical curves can be fitted to the experimental ones in order to determine the value of $L_{\|}$.

\section{Application to InSe.}

2.1 EXPERIMENTAL. - InSe is a lamellar semiconductor which can be obtained either as $\mathrm{p}$ or $\mathrm{n}$ type. Its structure consists of covalently bonded layers (Se-In-In-Se) held together by Van der Waals forces. Single crystals are grown by the nonstoichiometric Bridgman-Stockbarger method [29, 30]. n-type samples are $\mathrm{Cl}$ doped $\left(n \simeq 10^{15} / \mathrm{cm}^{3}\right) ;$ p-type samples are $\mathrm{Zn}$ doped $\left(p=10^{14} / \mathrm{cm}^{3}\right)$.

Electronic [20 and references therein] and optical $[21,31]$ properties are fairly well known. Table I summarizes the optical parameters which will be useful in the following.

The crystals we used were obtained from large ingots. // $c$ samples were cut with a surgical blade. This resulted in a bad surface which was characterized by far from ideal reflection conditions (the surface was dull). As we shall see later on, this fact is important to underline. $\perp c$ electrodes were obtained by gently cleaving the samples. The surface was smooth and shiny (good reflection conditions). Back ohmic contacts were established by ultrasonic soldering of indium metal on n-type crystals and with conductive silver paint on p-type ones. Crystals were encapsulated in a silicone rubber adhesive except for the face which was exposed to the electrolyte. The active area of the samples was a few $\mathrm{mm}^{2}$ large.

We have used a classical three electrode electrochemical set up. The reference electrode was a saturated calomel electrode (SCE) and the counter electrode a platinum wire. The cell, with a flat optical window, was made of quartz.

A parallel monochromatic light beam was obtained using an optical set-up in which a grating monochromator was included. The beam was mechanically chopped and the induced photocurrent was detected with a lock in amplifier. When needed, the sample could rotate around its median axis in order to vary the angle of incidence of the light. A polarizing filter was intercalated between the monochromator and the electrochemical cell so that either the ordinary ray or the extraordinary ray was illuminating the junction. The incident flux upon the samples was of the order of $10^{-6} \mathrm{~W} / \mathrm{cm}^{2}$.

Table I. - Optical properties of InSe.

\begin{tabular}{ccccc}
\hline$\lambda(\mu \mathrm{m})$ & 0.7 & 0.8 & 0.9 & 1 \\
\hline$\alpha_{x}(E \perp c) \mathrm{cm}^{-1}$ & 3230 & 2240 & 1850 & 1000 \\
\hline$\alpha_{z}(E / / c) \mathrm{cm}^{-1}$ & 58000 & 43000 & 35000 & 18000 \\
\hline$n_{x}(E \perp c)$ & 2.95 & 2.88 & 2.84 & 2.82 \\
\hline$n_{z}(E / / c)$ & 3.05 & 2.98 & 2.95 & 3.17 \\
\hline
\end{tabular}


As an electrolyte we used a $1 \mathrm{M} \mathrm{H}_{2} \mathrm{SO}_{4}$ solution which was prepared with distilled water.

The flat-band potential of InSe electrodes in an aqueous electrolyte cannot be evaluated precisely. Details of the measurements which were carried out, using three different methods, are largely reported in reference [18]. Finally, by analysing the photocurrent-potential characteristics of the junctions and the open-circuit potentials under illumination, the flatband potential of $n-I n S e$ was found to be close to $-0.6 \mathrm{~V} / \mathrm{SCE}$ and the one for $\mathrm{p}$-InSe close to $+0.5 \mathrm{~V} / \mathrm{SCE}$. These approximate values were confirmed by the evolution of the reverse dark current intensity with the redox potential and with the concentration of the species of the couple dissolved

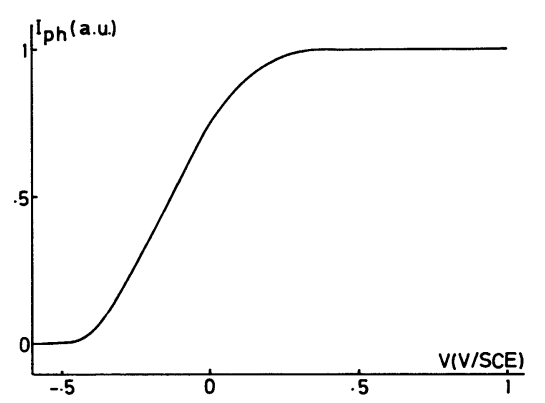

a)

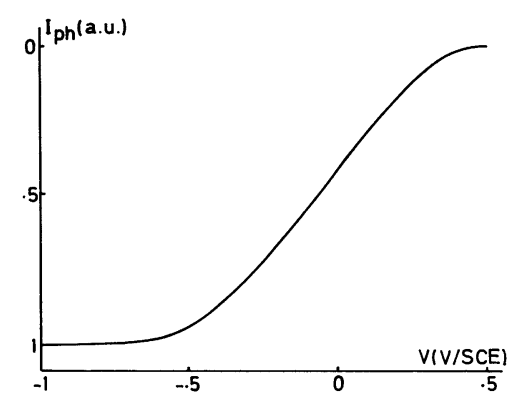

b)

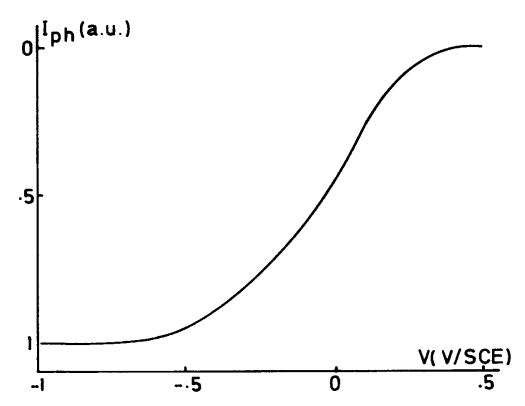

c)

Fig. 2. - Photocurrent-voltage characteristics of $\mathrm{InSe} / \mathrm{H}_{2} \mathrm{SO}_{4}$ junctions under a weak illumination ( $\simeq 10^{-6} \mathrm{~W} / \mathrm{cm}^{2}$ ). (a) n-type sample, $\perp c$ surface ; (b) $\mathrm{p}$ type sample, $\perp c$ surface; (c) p-type sample, // $c$ surface. in the electrolyte (injection of majority carriers from the solution towards the semiconductor for redox couples closes to the position of the majority carrier band at the interface [18]). In order for the Gärtner model to be valid, we applied a band bending of $\simeq 1.5 \mathrm{eV}$, that is a potential of $+1 \mathrm{~V} / \mathrm{SCE}$ for n-type crystals and $-1 \mathrm{~V} / \mathrm{SCE}$ for p-type ones. Typical photocurrent-voltage characteristics of InSe/ $\mathrm{H}_{2} \mathrm{SO}_{4} 1 \mathrm{M}$ junctions are shown in figures $2 \mathrm{a}$ ( $\mathrm{n}$ type,$\perp c), 2 \mathrm{~b}(\mathrm{p}$-type,$\perp c)$ and $2 \mathrm{c}$ (p-type, //c).

\subsection{RESULTS.}

2.2.1 // c electrodes. - This geometry permits the measurement of $L_{\perp}$ as described in the theoretical section. Experiments have been carried out with ptype electrodes. The reaction of reduction of protons permits the flow of photogenerated electrons into the electrolyte [18].

As mentioned above, the surface of the $/ / c$ electrodes is not perfect and, at least partially, diffuse reflection follows.

Two extreme cases may be considered :

(i) reflection is totally diffuse : thus, for a given wavelength, the reflection coefficient of the ordinary beam is equal to that of the extraordinary beam since, in that case, the reflection does not depend on the state of polarization of the incident light ;

(ii) despite bad surface conditions, the experimental reflection is identical to the theoretical reflection.

To avoid this gap between experiment and theory, we will consider the ratios of ratios $\left(J_{\mathrm{e}} / J_{\mathrm{o}}\right)_{\lambda_{1}} /$ $\left(J_{\mathrm{e}} / J_{\mathrm{o}}\right)_{\lambda_{2}}$ instead of the simple ratios $\left(J_{\mathrm{e}} / J_{\mathrm{o}}\right)_{\lambda}$.

Let :

$$
\begin{gathered}
\left(J_{\mathrm{e}} / J_{\mathrm{o}}\right)_{\lambda_{i}} \equiv \rho_{i} \quad(i=1 \text { or } 2) \\
J_{\mathrm{e} i}=\left(1-R_{\mathrm{e} i}\right) \xi_{\mathrm{e} i}\left(\alpha, L_{\perp}, W\right) \quad(\text { see } 1 \mathrm{a}) \\
J_{\mathrm{o} i}=\left(1-R_{\mathrm{o} i}\right) \xi_{\mathrm{o} i}\left(\alpha, L_{\perp}, W\right) \quad(\text { see } 1 \mathrm{~b}) .
\end{gathered}
$$

Then we have

$$
\rho_{1} / \rho_{2}=\frac{\left(1-R_{\mathrm{e} 1}\right)}{\left(1-R_{\mathrm{o} 1}\right)} \frac{\left(1-R_{\mathrm{o} 2}\right)}{\left(1-R_{\mathrm{e} 2}\right)} \frac{\xi_{\mathrm{e} 1} \xi_{\mathrm{o} 2}}{\xi_{\mathrm{o} 1} \xi_{\mathrm{e} 2}} .
$$

In case (i), $R_{\mathrm{o} i}=R_{\mathrm{e} i}$ and $R_{\mathrm{o} 2}=R_{\mathrm{e} 2}$ and (18) becomes

$$
\rho_{1} / \rho_{2}=\frac{\xi_{\mathrm{e} 1} \xi_{\mathrm{o} 2}}{\xi_{\mathrm{o} 1} \xi_{\mathrm{e} 2}}
$$

In case (ii) and considering relations (2a), (2b) and table I we have:

for $\lambda_{1}=1 \mu \mathrm{m}$ and $\lambda_{2}=0.7 \mu \mathrm{m}$,

$$
\frac{1-R_{\mathrm{e} 1}}{1-R_{\mathrm{o} 1}} \frac{1-R_{\mathrm{o} 2}}{1-R_{\mathrm{e} 2}}=0.973
$$


for $\lambda_{1}=0.9 \mu \mathrm{m}$ and $\lambda_{2}=0.7 \mu \mathrm{m}$,

$$
\frac{1-R_{\mathrm{e} 1}}{1-R_{\mathrm{o} 1}} \frac{1-R_{\mathrm{o} 2}}{1-R_{\mathrm{e} 2}}=1.001
$$

for $\lambda_{1}=0.8 \mu \mathrm{m}$ and $\lambda_{2}=0.7 \mu \mathrm{m}$,

$$
\frac{1-R_{\mathrm{e} 1}}{1-R_{\mathrm{o} 1}} \frac{1-R_{\mathrm{o} 2}}{1-R_{\mathrm{e} 2}}=1.003 \text {. }
$$

The real experimental situation is necessarily in the range between the extrema (i) and (ii). Hence, it can be considered that the experimental value $\rho_{1} / \rho_{2}$ which is measured is always such that

$$
\rho_{1} / \rho_{2}=\frac{\xi_{\mathrm{e} 1} \xi_{\mathrm{o} 2}}{\xi_{\mathrm{o} 1} \xi_{\mathrm{e} 2}} .
$$

Comparison between theory and experiment has been established on such a basis, taking for $\lambda_{2}$ the value of $0.7 \mu \mathrm{m}$. In figure 3 , it may be seen that a satisfactory fit of $\rho_{1} / \rho_{2}$ curves is obtained for an effective diffusion length $\perp c, L_{\perp}$, of $30 \mu \mathrm{m}$. Using photovoltaic spectra of In/InSe solid junctions Segura et al. [20,32] found a value of the order of $100 \mu \mathrm{m}$ for $L_{\perp}$, in n-type crystals. Ours, measured in p-type samples, is smaller by a factor of 3 , but, owing to the poor surface conditions of our electrode, a diffusion length of $30 \mu \mathrm{m}$ is, nevertheless, noteworthy.

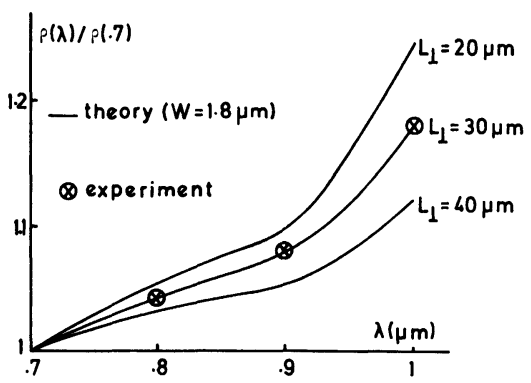

Fig. 3. - Comparison of theoretical and experimental values of the ratio $\rho_{1} / \rho_{2}$ '(p-type electrode, $\left./ / c\right)$.

2.2.2 $\perp$ c electrodes. - This configuration is used to measure $L_{\|}$.

As mentioned earlier, $\perp c$ electrodes are obtained by cleaving the samples and their surface is smooth. The experimental reflection is thus correctly described by the theoretical formulae and the ratios $J_{\mathrm{e}} / J_{\mathrm{o}}$ can be used directly. We have worked with both $\mathrm{n}$ and $\mathrm{p}$ type InSe.

* p-type electrodes :

for this type of material in an acidic electrolyte, the flow of minority carriers from the solid towards the electrolyte is absorbed by the reduction of protons. The electrode does not corrode and there is no interfacial film on the surface of the semiconductor. $J_{\mathrm{e}}$ and $J_{\mathrm{o}}$ can thus be calculated using relations (4), (5), (13) and (14) and table I for the values of the optical coefficients.

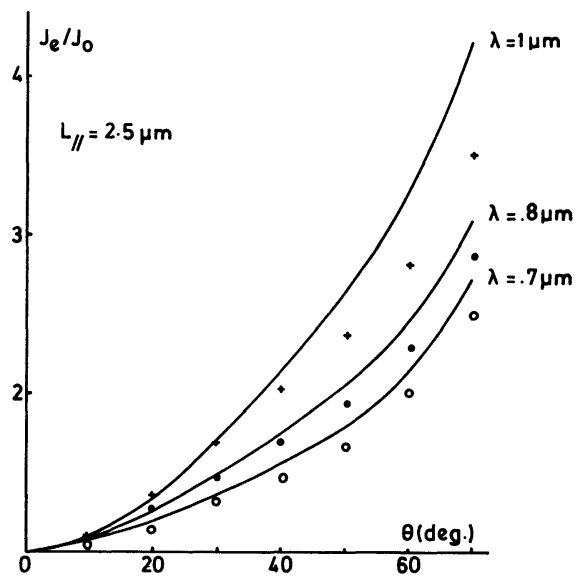

a)

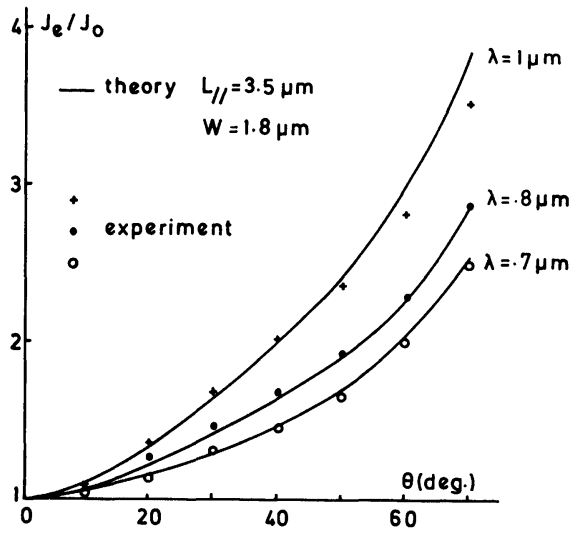

b)

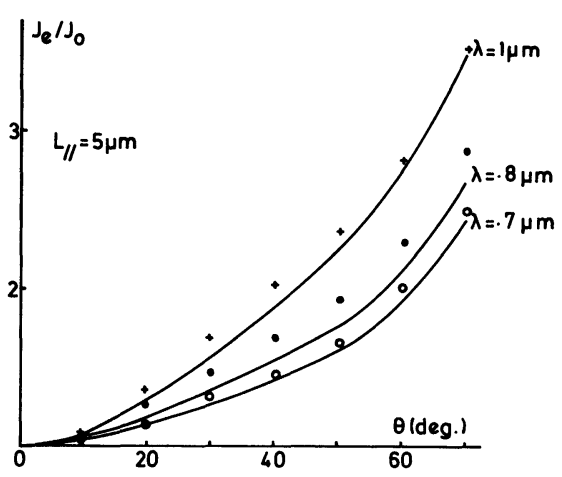

c)

Fig. 4. - Comparison of theoretical and experimental values of the ratio $J_{\mathrm{e}} / J_{\mathrm{o}}$ for a p-type electrode $(\perp c)$. (Theory : solid curves ( - ) experiment : (O) $\lambda=0.7 \mu \mathrm{m}$, (•) $\lambda=0.8 \mu \mathrm{m},(+) \lambda=1 \mu \mathrm{m}$.) (a) Theory with $L_{\|}=2.5 \mu \mathrm{m}$; (b) theory with $L_{\|}=3.5 \mu \mathrm{m}$; (c) theory with $L_{\|}=5 \mu \mathrm{m}$. 
A comparison between experimental and theoretical ratios $J_{\mathrm{e}} / J_{\mathrm{o}}$ is shown in figures $4 \mathrm{a}, \mathrm{b}$, c. It may be seen that a fairly good fit is obtained for $L_{\|}=$ $3.5 \mu \mathrm{m}$. The small divergence which appears for $\lambda=1 \mu \mathrm{m}$ at large angles is due to the dispersion of the monochromator. An effective diffusion length of $3.5 \mu \mathrm{m}$ is totally coherent with those previously published by Segura et al. [20], [32]. The more recent value which was obtained on p-type samples (by photomagnetoelectric measurement) is $4 \mu \mathrm{m}$ [33].

* n-type electrodes :

when a photocurrent is flowing, an n-type electrode corrodes. Hole flux at the surface of the semiconductor causes the following dissolution reaction to occur [18] :

$$
\mathrm{InSe}+3 \mathrm{~h} \rightarrow \mathrm{In}^{3+}+\mathrm{Se}
$$

and a selenium film grows on the sample. This film cannot be neglected in the present experiment because it modifies the conditions of light penetration into the InSe electrode. $\mathrm{A} \mathrm{H}_{2} \mathrm{O} / \mathrm{Se} / \mathrm{InSe}$ interface must be considered. The theoretical model must be adapted to this situation and must take into account the reflection at the $\mathrm{H}_{2} \mathrm{O} / \mathrm{Se}$ interface, the refraction of the light within the selenium film and the reflection at the $\mathrm{Se} / \mathrm{InSe}$ interface. In the range of wavelengths we used, selenium is transparent and its refraction index is known [34]; the reflection coefficients at both interfaces and the angle of refraction can be easily calculated by the Fresnel formula and the Descartes law respectively. Multiple reflection within the selenium film and interference effects are neglected.

When these modifications with respect to the original theory developed in the first section are taken into account, it can be seen in figures $5 \mathrm{a}, \mathrm{b}$ and c that a very satisfactory fit is found for $n$-type $\perp c$ electrodes when a diffusion length of $8 \mu \mathrm{m}$ is considered. Once again, this value is found to agree very well with the previous measurements $[20,32]$.

\section{Conclusion.}

The photoelectrochemical properties of lamellar semiconductors are greatly influenced by the optical birefringence of this family of materials. A theoretical model can be developped. It displays precisely the effects of the polarization of the incident light beam on the intensity of the photocurrent.

When comparing this model to experimental results, it is possible to determine the effective diffusion length of photogenerated carriers. This technique has been applied to the III-VI compound InSe : the results are totally coherent with previous measurements which were carried out by more

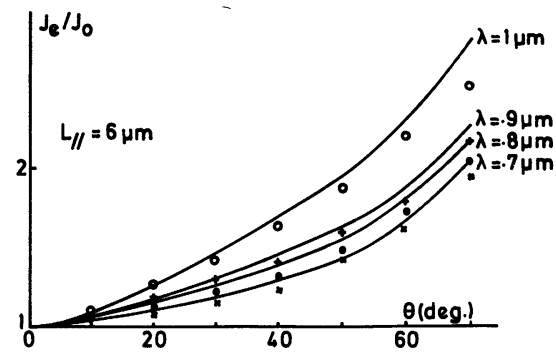

a)

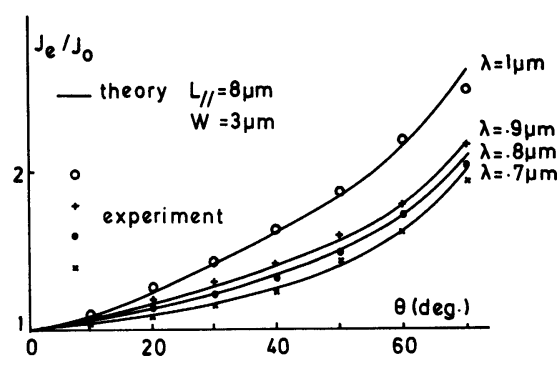

b)

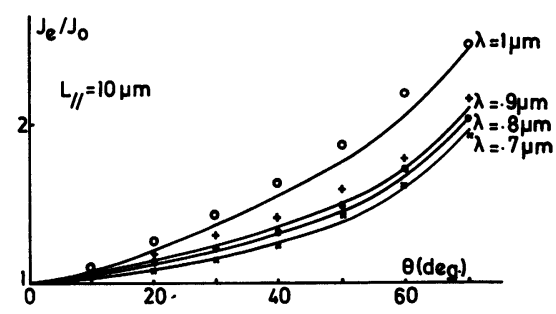

c)

Fig. 5. - Comparison of theoretical and experimental values of the ration $J_{\mathrm{e}} / J_{\mathrm{o}}$ for a $\mathrm{n}$ type electrode $(\perp c)$. (Theory: solid curves (-) experiment : (×) $\lambda=$ $0.7 \mu \mathrm{m},(\bullet) \lambda=0.8 \mu \mathrm{m},(+) \lambda=0.9 \mu \mathrm{m}$, (O) $\lambda=$ $1 \mu \mathrm{m}$.) (a) Theory with $L_{\mathrm{I}}=6 \mu \mathrm{m}$; (b) theory with $L_{\|}=8 \mu \mathrm{m}$; (c) theory with $L_{1}=10 \mu \mathrm{m}$.

conventional means. This confirms the ability of our method which has the advantage that it does not necessitate any previous treatment of the sample. Indeed, electrolytic junctions are rather easily obtained.

Finally, we point out another potential application of the described model : it may be a way to measure the flat band potential $V_{\mathrm{fb}}$ of a semiconducting electrode. Indeed, the space-charge width, $W$, of the junction appears in the relations giving the intensity of the different photocurrents. Thus, this value $W$ may also be found by fitting experimental and theoretical curves of the ratio $J_{\mathrm{e}} / J_{\mathrm{o}}$. If $W$ is known for a given polarization $V$ of the electrode, it is therefore possible to know the band-bending $V$ - 
$V_{\mathrm{fb}}$ (and thus $V_{\mathrm{fb}}$ ) if $W$ follows the Mott-Schottky law $\left(W \propto\left(V-V_{\mathrm{fb}}\right)^{1 / 2}\right.$ [35]). Unfortunatly, for InSe/electrolyte junctions this law is not verified [18] and we have not been able to test this latter application.

\section{Acknowledgments.}

The author is grateful to J. M. Besson for helpful discussions and to A. Chevy for providing the crystals.

\section{References}

[1] Goodman, A. M., J. Appl. Phys. 32 (1961) 2550.

[2] Goodman, A. M., Goodman, L. A. and GossenBerger, H. F., R.C.A. Rev. 44 (1983) 326.

[3] Annual Book of A.S.T.M. Standards, F.391, Part 43 (Philadelphia) 1979, pp. 770-778.

[4] Schwarz, R. and Matthey, C., Helv. Phys. Acta 57 (1984) 687.

[5] Agarwala, A., Tewary, V. K., Agarwal, S. K. and JAIN, S. C., Solid State Electron. 23 (1980) 1021.

[6] Arora, N. D., Chamberlain, S. G. and Rouston, D. L., Appl. Phys. Lett. 37 (1980) 325.

[7] Bourée, J. E., Le Quang NAM, Barbé, M., Perraki-Goutsou, V. and Rodot, M., Proc. 6th E.C. Photovoltaic Solar Energy Conf. (D. Reidel Dordrecht) 1985 , p. 1051.

[8] Chiang, C. L., Agner, S. and Ballman, A. A., Appl. Phys. Lett. 43 (1982) 1113.

[9] Moore, A. A., Appl. Phys. Lett. 40 (1982) 403.

[10] Gautron, J., Lemasson, P., J. Cryst. Growth 59 (1982) 332.

[11] Chari, A., De Mierry, P., Menik, A. and AUCOUturier, M., Rev. Phys. Appl. 22 (1987) 655.

[12] Physics and Chemistry of Materials with layered structures (D. Reidel, Dordrecht, Boston) Vol. I-VI (1977-1979).

[13] Gerischer, H., J. Electroanal. Chem. 150 (1983) 553 and references therein.

[14] LeVy-Clément, C. and Theys, B., J. Electrochem. Soc. 131 (1984) 1300.

[15] Douay, V. and Gorochov, O., J. Chim. Phys. 83 (1986) 247.

[16] McEvoy, A. J., Etmam, M. and Memming, R., $J$. Electroanal. Chem. 190 (1985) 225.

[17] Fotouhi, B., KatTy, A. and Gorochov, O., $J$. Electrochem. Soc. 132 (1985) 2181.
[18] ThEYs, B., Thèse d'Etat, Université Paris VII (1987).

[19] Kautek, W., Decker, F., Gerischer, H., Phys. Stat. Sol. (b) 122 (1984) 651.

[20] Segura, A., Guesdon, J. P., Besson, J. M. and Chevy, A., J. Appl. Phys. 54 (1983) 876.

[21] Piccioli, N., Le Toullec, R., Bertrand, F. and Chervin, J. C., J. Phys. France 42 (1981) 1129.

[22] Gartner, W. W., Phys. Rev. 116 (1959) 84.

[23] See for example Hartshorne, N. H., STUART, A., Practical optical crystallography (Edward Arnold Ltd, London) 1964.

[24] Born, M. and Wolf, E., Principles of Optics, 6th edition (Pergamon Press, Oxford) 1980.

[25] Mosteller, L. P., Jr and Wooten, W., J. Opt. Soc. Am. 58 (1968) 511.

[26] Bruhat, G., Optique 6th edition (Masson et Cie, Paris) 1965.

[27] Greenaway, D. L., Harbeke, C., Bassani, F. and Tosatti, E., Phys. Rev. 178 (1969) 1340.

[28] Mathieu, J. P., Optique (Société d'Edition d'Enseignement Supérieur, Paris) tome I, 1965.

[29] Chevy, A., Kuhn, A. and Martin, M. S., J. Cryst. Growth 38 (1977) 118.

[30] Chevy, A., Thèse d'Etat, Université Paris VI (1981).

[31] Le Toullec, R., Private Communication.

[32] Segura, A., Thèse de $3^{\mathrm{e}}$ cycle, Université Paris VI (1977).

[33] Cantarero, A., Pomer, F., Segura, A., Lloret, J. J. and Chevy, A., An. Fis. B 80 (1984) 16.

[34] Koehler, W. F., Odencrantz, F. K. and White, J. C., J. Opt. Soc. Am. 49 (1959) 109.

[35] For example MORRISON, S. R., Electrochemistry at semiconductor and oxydized metal electrodes (Plenum Press, New York) 1980. 